\title{
ConectAEE: Um Sistema de Fomento à Comunicação Multidisciplinar na Perspectiva da Educação Especial
}

\author{
Eberson Santos ${ }^{1}$, Igor Vanderlei ${ }^{1}$, Mariel Andrade ${ }^{1}$, Rodrigo Rocha ${ }^{1}$, Jean Araujo ${ }^{1}$ \\ ${ }^{1}$ Universidade Federal do Agreste de Pernambuco (UFAPE) \\ Av. Bom Pastor, s/n - Boa Vista - 55292-270 - Garanhuns - PE - Brasil \\ \{eberson.santos1, igor.vanderlei, marieljpa, rgcrocha, \\ teixeirajean1\}@gmail.com
}

\begin{abstract}
Several applications make it possible to follow the routine of students attending regular education only, but usually do not take into account the specificities of the target audience of Special Education. This paper presents ConectAEE, a Web system that allows a shared service to special education students, promoting communication among their teachers, parents and health professionals. Scrum was adopted, as development methodology, besides interviews and bibliographic study that allowed modeling the system. ConectAEE was evaluated through a focus group interview, which indicated that the system has the potential to become an ally in multidisciplinary educational actions.
\end{abstract}

Resumo. Diversas aplicações permitem acompanhar a rotina de alunos que frequentam exclusivamente o ensino regular, mas normalmente não consideram as especificidades relativas ao público-alvo da Educação Especial. Este artigo apresenta o ConectAEE, um sistema Web que permite um atendimento compartilhado aos discentes do ensino especial e promove a comunicação entre seus professores, pais e profissionais de saúde. Como metodologia de desenvolvimento, adotou-se o Scrum, além de entrevistas e estudo bibliográfico que permitiram modelar o sistema. O ConectAEE foi avaliado por meio de entrevista de grupo focal, que indicou que o sistema tem potencial para se tornar um aliado em ações educacionais multidisciplinares.

\section{Introdução}

A Educação Especial é o ramo da Educação encarregada por desempenhar, nos diversos níveis, modalidades e etapas do ensino, o Atendimento Educacional Especializado (AEE) para pessoas com deficiência (visual, auditiva, física etc.), transtornos globais de desenvolvimento (desordens do espectro autista) e altas habilidades ou superdotação [MEC 2008]. A função do AEE é considerar as necessidades específicas dos alunos e algumas das atividades oferecidas são o ensino do código Braille, da Língua Brasileira de Sinais (LIBRAS) e instrução e uso de tecnologia assistiva, que são dispositivos e práticas que proporcionam maior qualidade de vida aos indivíduos.

As diretrizes do Ministério da Educação (MEC) ressaltam que o AEE deve ser realizado preferencialmente na mesma escola em que o aluno está matriculado, podendo também ser ofertado em outra de ensino regular ou em centros de atendimento educacional especializado públicos ou privados [MEC 2008]. O Censo da Educação Básica de 2019 [INEP 2020], mostra que dentre os alunos público-alvo da Educação Especial que têm de 4 a 17 anos de idade e estão matriculados em classes comuns, 
40,8\% têm acesso às turmas de AEE. Ainda segundo o Censo, este percentual era de $37,4 \%$ em 2015. Diante disso, observa-se que mesmo que o avanço rumo à inclusão seja lento, uma grande parcela dessa população recebe de forma simultânea os ensinos regular e especial.

Outro dado relevante indica que um relacionamento ativo entre família e escola oferece ao estudante diversas vantagens, tais como a diminuição de problemas de comportamento, melhor rendimento escolar e maior frequência nas aulas [Saraiva e Wagner 2013]. Além disso, estudos evidenciam que terapeutas ocupacionais, fonoaudiólogos, fisioterapeutas e outros profissionais são responsáveis por beneficiarem a realização de atividades pedagógicas ao ocasionarem progressos na linguagem oral, desenvolvimento sensorial e motor de várias crianças e adolescentes [Melo e Ferreira 2009], [Pelosi e Nunes 2011].

À vista disso, a comunicação entre pais, especialistas da área de saúde e professores dos ensinos especial e regular pode impactar em uma melhoria do atendimento pedagógico prestado aos alunos do AEE. No entanto, esta comunicação é problemática porque há a diferença nas abordagens do ensino regular e especial e o fato de que, por vezes, o ensino especial, o ensino regular e o atendimento do profissional de saúde são realizados em escolas/instituições distintas, que podem estar localizadas em uma distância considerável uma da outra.

Diante desses desafios, o objetivo deste artigo é apresentar um sistema, denominado ConectAEE, que permite aos atores pertencentes às instituições da família, escola e saúde desfrutarem de um mesmo canal de comunicação, no qual ocorre a troca de informações do que está sendo vivenciado pelo aluno com deficiência, na sala de aula regular, na sala de atendimento especializado e/ou no consultório, além do acompanhamento de seu progresso. Diante disso, espera-se um alinhamento das práticas pedagógicas e clínicas realizadas com o aluno, maximizando assim, seu desenvolvimento e consequentemente sua inclusão.

Este artigo está organizado da seguinte maneira: a Seção 2 apresenta aspectos da educação inclusiva; Por sua vez a Seção 3 descreve a metodologia adotada; Em seguida na Seção 4 são apresentadas as principais funcionalidades do sistema proposto; A Seção 5 discute os resultados; Por fim, a Seção 6 apresenta as considerações finais e alguns trabalhos futuros.

\section{Educação Inclusiva}

O modelo de educação inclusiva surgiu nos anos 90 e é pautado no reconhecimento da existência de um conjunto de diferenças (físicas, culturais, etc.) que caracterizam a diversidade humana e na admissão que o sistema educacional deve adequar-se a elas [Miranda 2008]. Nesse sentido, as instituições de ensino devem se mostrar aptas a proporem projetos político-pedagógicos que contemplem esta diversidade.

Diante disso, Vilaronga e Mendes (2014) afirmam que educadores que trabalham individualmente nas salas de aula regulares sentem dificuldade para realizar ações pedagógicas de qualidade para os alunos atendidos pelo AEE. Para que o modelo inclusivo funcione, é necessário que haja um ensino colaborativo entre os professores das modalidades de ensino regular e especial, de forma que seja possível planejar e instruir ações para um grupo heterogêneo de estudantes.

Ainda na perspectiva da educação inclusiva, é válida a existência de uma rede 
de apoio composta por sujeitos que possam compartilhar métodos, técnicas e atividades que favoreçam o sucesso de professores e alunos [Pelosi e Nunes 2011]. Tal rede de apoio deve possuir caráter multidisciplinar, onde as particularidades do conhecimento de cada especialista sejam aplicadas de forma a atingir um objetivo em comum.

Na pesquisa conduzida por Silva et al. (2016), professores expressaram interesse em compreender alunos com deficiência e/ou transtorno global do desenvolvimento de um ponto de vista clínico, com o intuito de adequar materiais e métodos pedagógicos. Isso demonstra o quanto se faz necessário para os professores conhecerem, através de outro ângulo, os progressos e dificuldades de seus alunos e assim poderem traçar uma estratégia educacional adequada.

Para Polonia e Dessen (2005) a família e a escola são duas instituições igualmente relevantes quando se trata de atuar no crescimento físico, intelectual e pessoal dos estudantes. Considera-se que estas duas instituições também devem assumir a responsabilidade de agir de forma conjunta e cooperativa para alcançar os melhores resultados no desenvolvimento dos educandos.

Dessa forma, é essencial a presença de uma comunicação adequada entre todos os profissionais que estão envolvidos com um aluno frequentador do AEE para garantir que seu desenvolvimento seja apropriado e que não haja uma fragilidade em torno da Educação Especial. Para isso, podemos contar com o auxílio das Tecnologias da Informação e Comunicação (TIC) que proporcionam uma gama de recursos que têm revolucionado as formas de comunicação e servem como ferramenta para diminuir as barreiras no âmbito da aprendizagem [Araújo 2013].

\section{Metodologia}

O processo de software é uma abordagem sistemática que consiste em uma sequência de atividades que leva à produção de um sistema [Sommerville 2011]. Assim, as quatro principais atividades do desenvolvimento da ferramenta que culminaram neste trabalho foram: análise do problema, consolidação, desenvolvimento e avaliação do software.

A primeira fase foi a investigação do problema, na qual foi realizado um estudo da literatura sobre o tema de forma a construir uma base teórica sólida a respeito da Educação Especial. Também foi conduzida uma entrevista não estruturada com docentes especialistas na área de Educação Especial, assim como outros que atuam na rede municipal e estadual de ensino em Garanhuns - PE, com o intuito de coletar dados sobre como o problema da comunicação se apresenta na prática. As informações levantadas na revisão bibliográfica e na entrevista foram organizadas e deram origem aos requisitos do sistema que foram documentados na forma de histórias de usuário e classificados de acordo com a prioridade de desenvolvimento.

Levando em consideração a lista de funcionalidades que deveriam ser desenvolvidas para o sistema, ou seja, o backlog do produto levantado na etapa anterior, a segunda etapa preocupou-se em consolidar o entendimento a respeito de tais requisitos. Dessa maneira, foi idealizado o protótipo da interface e o Modelo Entidade Relacionamento (MER) da solução proposta. Esses aparatos serviram para estruturar o projeto e guiar sua construção. Para a confecção do protótipo, foi utilizado o Balsamiq Cloud $^{1}$, que é uma ferramenta gráfica para esboçar interfaces de usuário de sites e sistemas para diferentes plataformas. $O$ modelo de dados conceitual escolhido foi $o$ Modelo Entidade Relacionamento (MER), que pode especificar, de modo sistemático,

1 Disponível em: https://balsamiq.cloud/ 
as necessidades de dados dos usuários (Silberschatz et al. 2012).

Com o entendimento do sistema consolidado, partiu-se para a próxima fase: o desenvolvimento. O software foi desenvolvido para Web devido à mobilidade e portabilidade proporcionada por este tipo de arquitetura. Foi utilizada a linguagem de programação $\mathrm{PHP}^{2}$ sob o framework $\mathrm{Laravel}^{3}$, que se destaca por fornecer recursos concisos que auxiliam os desenvolvedores a escreverem códigos simples e de fácil manutenção [Stauffer 2019]. Para o banco de dados, foi utilizado o PostgreSQL ${ }^{4}$ que é um sistema de banco de dados objeto-relacional de código aberto. No contexto do desenvolvimento de software, Soares (2004) afirma que as metodologias ágeis são adequadas para projetos em que a equipe é pequena, o desenvolvimento rápido é fundamental e os requisitos podem sofrer alterações no decorrer do tempo. Levando em consideração as características do projeto proposto, optou-se pela adoção da metodologia ágil denominada Scrum, que funciona de forma incremental e iterativa e acarreta benefícios como um ritmo constante de desenvolvimento e controle do andamento do projeto [Schwaber 2004][Ferreira e Canedo 2019].

Com o sistema desenvolvido, a última etapa executada foi a avaliação. Para isso, foi necessário fazer uma análise da opinião de especialistas a respeito da proposta e aspectos do ConectAEE, através da técnica de coleta e análise de dados denominada grupo focal. Esta técnica consiste na reunião de um grupo de participantes previamente selecionados que podem dialogar entre si e trocarem ideias, obtendo ao fim resultados que ultrapassam a soma das partes individuais [Dias 2000].

\section{Sistema ConectAEE}

O principal intuito do ConectAEE é promover a comunicação entre professores do ensino regular e especial, familiares e outros profissionais que prestem qualquer tipo de atendimento ao aluno com deficiência, fornecendo funcionalidades importantes que podem tornar o processo de ensino multidisciplinar e compartilhado. A plataforma é livre, gratuita e foi projetada para o uso da comunidade e está disponível para os usuários no endereço <http: / / app.uag.ufrpe.br/conectaee> e seu código-fonte foi disponibilizado em <https://github.com/lmts-ufape/conectaee>.

$\mathrm{O}$ acesso ao sistema e às suas funcionalidades é efetivado após o cadastramento de um usuário. Depois disso, o ConectAEE oferece a possibilidade de gerenciar instituições de ensino. O cadastro de mais de uma instituição reproduz o contexto do mundo real, no qual os alunos da Educação Especial podem estar alocados, em turnos diferentes, em mais de uma escola ou centro de atendimento.

Para tornar a experiência com as funcionalidades do sistema mais proveitosa é necessário fazer o cadastro de alunos, informando suas identificações, instituições onde são atendidos, endereços, dados médicos e outras observações que o usuário achar pertinente. Na Figura 1 é apresentada a página do perfil de um aluno criado com dados fictícios.

O professor de AEE ou responsável que cadastra um aluno se torna automaticamente administrador do perfil registrado. Desta forma, eles podem autorizar e desautorizar o acesso de outros usuários, (professores do ensino regular, profissionais de saúde, outros responsáveis), assim como gerenciar quem exerce a função de

2 Disponível em: https://www.php.net/

3 Disponível em: https://laravel.com/

4 Disponível em: https://www.postgresql.org/ 
administrador deste perfil.

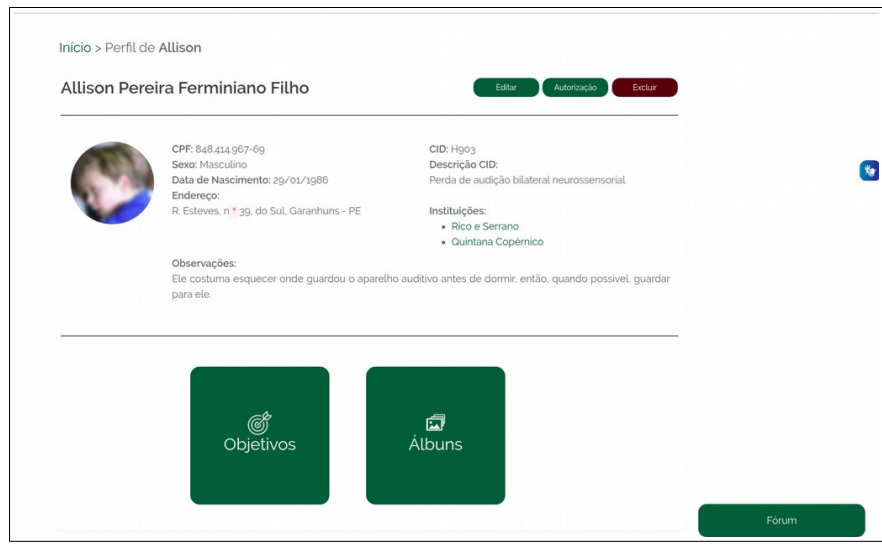

Figura 1. Interface para gerenciamento de aluno (dados fictícios)

Usuários que gerenciam o perfil de um aluno estão habilitados a criarem álbuns de imagens para serem compartilhados com os outros usuários envolvidos. Estes álbuns podem funcionar como espaço para guardar fotos de atividades desenvolvidas com os alunos na escola ou em outros ambientes, possibilitando aos familiares uma forma de manter um acervo visual de tais momentos.

O ConectAEE conta com um canal de comunicação específico para cada aluno cadastrado, como apresentado na Figura 2. Esse espaço, intitulado fórum, permite aos atores que possuem acesso ao perfil de um aluno enviar mensagens multimídia, que podem conter textos, imagens, vídeos e links, e proporciona uma maneira prática para deixar recados, esclarecer dúvidas, pedir opiniões e assim por diante.

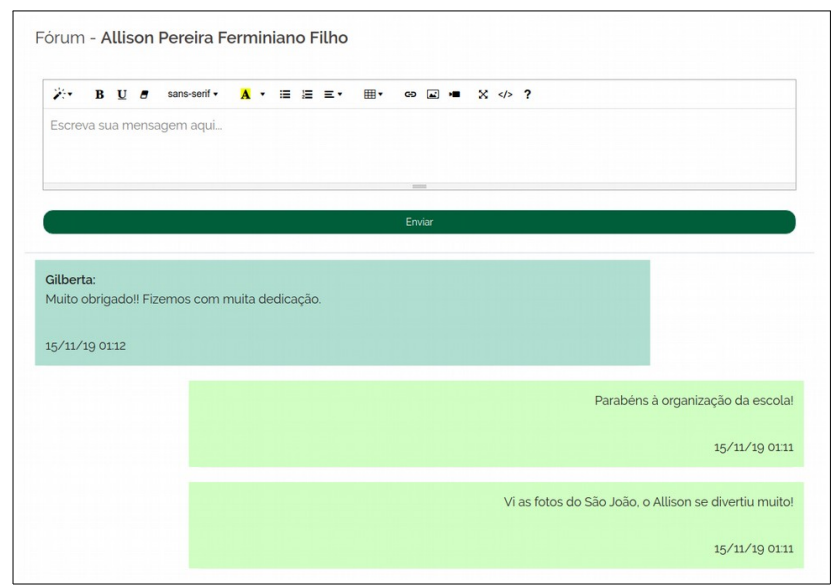

Figura 2. Fórum de um aluno (dados fictícios)

A principal funcionalidade do sistema diz respeito ao registro e gerenciamento dos objetivos almejados para os alunos. O educador ou profissional de saúde, que tenha acesso ao perfil de um aluno, pode descrever e atribuir objetivos educacionais ou relativos a saúde para ele. Os dados de um objetivo (descrição, prioridade, histórico da situação) ficam disponíveis para consulta por todos os envolvidos com o discente em questão. Como exemplo, a Figura 3 apresenta objetivos criados por dois tipos de usuários diferentes. 


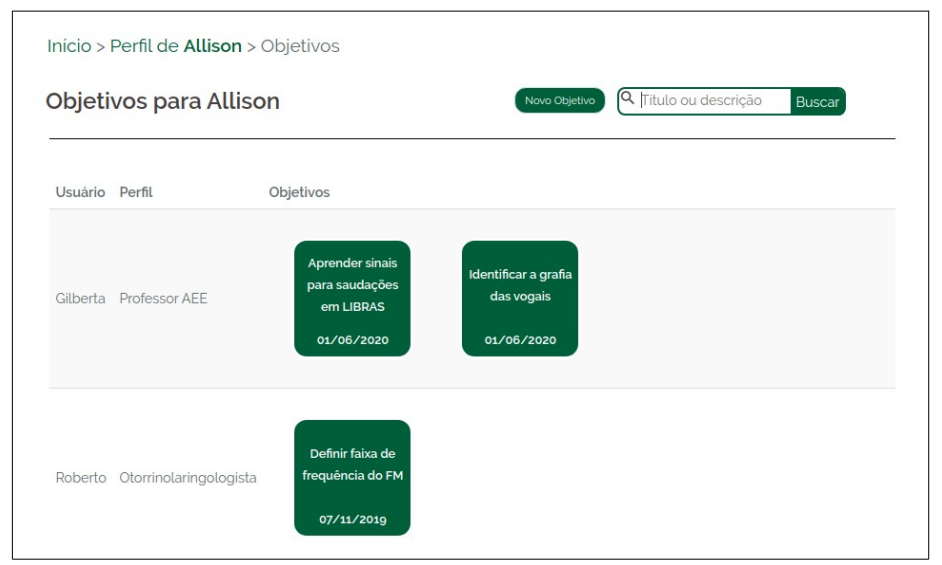

Figura 3. Objetivos para um aluno (dados fictícios)

O usuário que cadastra um objetivo pode planejar as atividades que serão desenvolvidas para atingi-lo. Tais atividades podem ser consideradas como metas que possuem um escopo menor e mais simples, que quando realizadas em conjunto culminarão na realização do objetivo. Ademais, para estimular a participação multidisciplinar da equipe que acessa o perfil do aluno, cada usuário pode enviar sugestões de atividades para aquele objetivo, baseados em suas próprias experiências e conhecimentos específicos. Na Figura 4 é apresentada a interface contendo as atividades e sugestões na visão do criador de um objetivo.

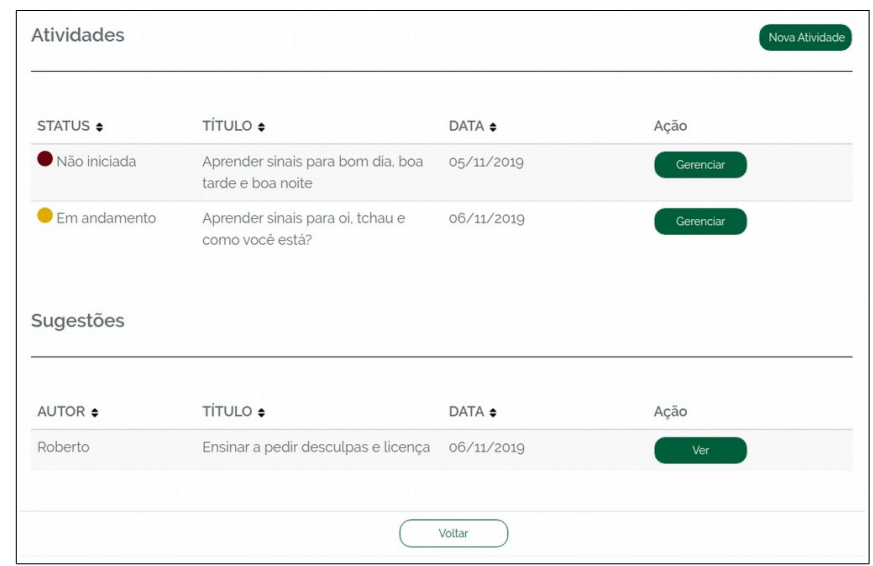

Figura 4. Atividades e sugestões para um objetivo (dados fictícios)

O intuito de dispor objetivos, atividades e sugestões de maneira compartilhada é fomentar a comunicação e a colaboração entre os atores. Além disso, é possível manter um histórico dos atendimentos já realizados por profissionais diversos que pode ser consultado a qualquer momento. Por fim, todos podem acompanhar e contribuir com o desenvolvimento do aluno e potencialmente alcançar resultados melhores.

O ConectAEE é responsivo, ou seja, adapta seu conteúdo para diferentes dispositivos e tamanhos de telas. Para garantir o sucesso na utilização do software, foram consideradas medidas para melhorar sua acessibilidade, como a implantação de modo de alto-contraste para beneficiar indivíduos com baixa visão e a utilização da ferramenta VLibras ${ }^{5}$ para que pessoas surdas possam interagir com o software. 


\section{Resultados e Discussão}

Para avaliação da proposta, foi realizada uma entrevista de grupo focal com o intuito de avaliar qualitativamente a aplicação. Os objetivos da avaliação foram averiguar se o ConectAEE é adequado para o fomento à comunicação no contexto da Educação Especial e identificar problemas e possíveis melhorias no sistema. Infelizmente, ainda não foi possível obter um resultado experimental relativo à validação do sistema, uma vez que o planejamento para implantação da ferramenta foi adiado por tempo indeterminado devido à pandemia de covid-19.

Os participantes da entrevista de grupo focal estão descritos abaixo:

- P1 - Supervisor da Secretaria de Educação do município de Garanhuns-PE;

- P2 - Instrutora de LIBRAS;

- P3 - Doutora em Educação Especial e professora aposentada de pessoas surdas;

- P4 - Doutor em Educação e professor do curso de Licenciatura em Pedagogia da UFAPE.

Uma restrição encontrada na avaliação do sistema diz respeito à presença de profissionais de saúde, assim como pais ou responsáveis de alunos com deficiência na execução da entrevista de grupo focal. Sendo assim, a discussão que se segue é apresentada sob a ótica de sujeitos que possuem perfis voltados à Educação.

A entrevista transcorreu tendo como instrumento norteador um roteiro de entrevista semiestruturada elaborado na fase de planejamento do grupo focal. A reunião foi gravada em áudio, mas considerando a restrição de espaço deste artigo, as respostas dos quatro participantes não são exibidas em sua totalidade e apenas recortes dos diálogos que apresentam conteúdo concernente às perguntas abordadas e que representam o entendimento geral dos entrevistados são expostos a seguir.

\section{Pergunta 1. A interface do sistema está amigável?}

P3 - Eu não sou muito da informática [...], mas a gente consegue perceber que é uma coisa que não é complicada, é prático.

P1 - O layout é bem simples, é muito intuitivo! [...] É bem claro também né, os informes pra fazer todos os cadastros, fazer as inserções... São bem óbvias, não tem informação excedente, não tem nada que vai encher linguiça [...] a gente que lida muito com informática já, a gente vai ter muita facilidade.

O propósito desta questão foi identificar a percepção que os entrevistados tiveram sobre os elementos gráficos disponíveis no ConectAEE. Neste ponto, foi relatado a simplicidade e clareza da interface, entretanto houve uma certa preocupação com usuários que não têm familiaridade com recursos computacionais, levantando a possibilidade de serem feitos tutoriais e capacitações para os professores se habituarem à ferramenta. usuários?

Pergunta 2. O software tem potencial para atender as demandas dos seus

P2 - [...] esse sistema é um sonho da educação inclusiva [...] quem trabalha na Sala de Recursos sempre é cobrado esses dados. Como o aluno está se desenvolvendo, quais foram as atividades, é... Se houve um avanço ou houve a regressão em um 
processo. E nem sempre temos essa disponibilidade de transporte pra ir até a escola e passar isso pessoalmente para professora do ensino regular e a professora também nos dar esse retorno se a nossa prática tá fazendo efeito, tá surtindo efeito na escola.

P3 - [...] para o professor que tá lá na sala de aula eu acho que isso vai ser uma mão na roda, uma praticidade muito grande.

Neste ponto, buscou-se verificar se as funcionalidades que o software oferece são, de fato, úteis para o público ao qual elas se destinam. De modo geral, os participantes acreditam que o software atende as demandas dos usuários e relataram que o sistema contribui para tornar o acompanhamento dos alunos mais prático e colaborativo.

\section{Pergunta 3. Como vocês avaliam a recepção do público a quem o software se destina?}

P3 - Eu acho que vai requerer um tempo de ajuste.

P4 - Depois fica muito mais fácil, [...] as pessoas ainda não têm o domínio, não conhecem, os ajustes, tudo isso, mas acho que quando ele entrar que baixar a poeira vai ser muito interessante.

P1 - Agora, no início realmente vai ter essa fase de transição, de adaptação.

Ao contrário da hipótese pressuposta de que os usuários receberiam o sistema com satisfação devido à praticidade proporcionada, os entrevistados problematizaram que eles poderiam sofrer com um período de adaptação, considerando principalmente as pessoas que preferem conteúdo impresso em detrimento ao digital. Entretanto, mesmo com essa fase de ajustes, os entrevistados acreditam que estes usuários perceberão os benefícios que o ConectAEE proporciona.

Pergunta 4. Quais consequências a implantação desse sistema acarretaria ao ensino do público-alvo do AEE?

$P 2$ - O professor do AEE fica com essa responsabilidade de fazer com que o aluno se desenvolva não na parte cognitiva né, disciplinar, mas na autonomia. E aí, até nesse compartilhamento dessas atividades o professor da sala regular vai ver o que a professora né, da sala de recursos vai tá fazendo: "Ah, então eu posso acrescentar tal atividade" [...]

P4 - Acho que é isso, ratifico aí. A partir do momento que tem trabalho em equipe pela praticidade ai do programa e tudo, quem ganha é o aluno, o professor ganha também.

A partir do relato dos entrevistados, podemos afirmar que o ConectAEE tem o potencial de tornar o ensino multidisciplinar e facilitar o trabalho dos professores, que poderão contar com o auxílio de outros profissionais para realizar suas funções.

\section{Pergunta 5. Como os alunos podem ser afetados pelo ConectAEE?}

P1 - Essa integração, ela vai possibilitar melhorar e maximizar as ações, uma vez que todas as ações estariam ali, todo o acompanhamento que vai ser realizado estaria ali.

P2 - [...] é muito importante, esse alinhamento. Porque a professora da sala do AEE tá trabalhando, a professora do ensino regular ela pode também complementar, dar continuidade, o profissional de apoio também, alguns tem profissional de apoio, 
então vai, com certeza, contribuir e alinhar esse atendimento, vai ampliar o atendimento que era só na Sala de Recurso, a professora regular vai ter acesso, o pai também e todos os envolvidos.

No entendimento dos participantes do grupo focal, o ConectAEE possui a capacidade de exercer um papel no desenvolvimento dos discentes porque propicia o alinhamento das atividades desempenhadas com eles. Os entrevistados relataram que uma vez que a informação esteja disponível de forma síncrona para os diversos agentes envolvidos, cada um pode atuar de maneira a complementar o trabalho do outro e assim maximizar o aprendizado do aluno.

De maneira geral, foi apontado, através da análise dos resultados, que o sistema pode contribuir positivamente para o processo de acompanhamento dos alunos que são amparados pelo AEE, bem como no desenvolvimento deles, ao facilitar a comunicação entre todos os envolvidos neste contexto.

\section{Considerações Finais}

A literatura mostra que há um problema na comunicação entre os diversos agentes envolvidos no contexto da Educação Especial, ou seja, professores (ensinos regular e especial), profissionais da área da Saúde e pais/responsáveis. Apesar da atuação de uma equipe multidisciplinar causar efeitos positivos na aprendizagem de pessoas com deficiência, fatores como distância e tempo impedem este processo.

A inserção das TIC neste cenário tem potencial para se tornar um forte aliado no auxílio e estímulo das práticas pedagógicas e clínicas conjuntas, beneficiando, consequentemente aos alunos/pacientes em questão. Entretanto, até o presente trabalho não foram encontradas ferramentas com este propósito específico, havendo apenas softwares que se restringem à sala de aula regular e que não dão suporte aos alunos que são atendidos pelo AEE ou ferramentas de comunicação de propósito geral como é o caso das redes sociais.

Em virtude disto, este trabalho apresenta um sistema de informação Web, o ConectAEE, que é uma plataforma que permite aos usuários acompanhar, de forma individual, o atendimento planejado para os alunos do AEE e mensurar o progresso deles. Como forma de avaliação do sistema, realizou-se uma entrevista de grupo focal que permitiu confirmar que a ferramenta tem potencial para estimular a comunicação e processo de ensino dos profissionais, na aprendizagem dos alunos público-alvo do AEE e na supervisão por parte dos pais ou responsáveis.

Entretanto, algumas limitações do sistema foram encontradas. Uma delas é que instituições de ensino não podem se cadastrar como usuárias da plataforma, o que auxiliaria na confirmação da identidade dos professores cadastrados. A segunda restrição diz respeito a falta de emissão de relatórios que podem contribuir com a quantificação de dados sobre a Educação Especial dos municípios brasileiros. Os trabalhos futuros indicam o desenvolvimento de uma versão que elimine as limitações supracitadas da ferramenta e a realização de um estudo de caso onde o ConectAEE seja implantado em uma instituição de ensino para constatar os benefícios de sua utilização.

\section{Referências}

Araújo, A. L. S. O. de, Brito, R. R. de e Silva, A. P. da (2013). Softwares para educação inclusiva: uma revisão sistemática no contexto de SBIE e WIE. Brazilian 
Symposium on Computers in Education (Simpósio Brasileiro de Informática na Educação - SBIE), 24(1), 507

Dias, C. A. (2000). GRUPO FOCAL: técnica de coleta de dados em pesquisas qualitativas. Informação \& Sociedade, v. 10, n. 2, p. 12.

Ferreira, V. e Canedo, E. (2019). Autenticidade na aprendizagem baseada em projetos para desenvolvimento de software: Uma Revisão Sistemática de Literatura. Brazilian Symposium on Computers in Education (Simpósio Brasileiro de Informática na Educação - SBIE), 30(1), 41.

INEP. Instituto Nacional de Estudos e Pesquisas Educacionais Anísio Teixeira (2020). Resumo Técnico: Censo da Educação Básica 2019. Brasília, DF: Inep.

MEC. Ministério da Educação (2008). Diretrizes operacionais da Educação Especial para o Atendimento Educacional Especializado na Educação Básica. Brasília, DF.

Melo, F. R. L. V. de e Ferreira, C. C. DE A. (2009). O cuidar do aluno com deficiência física na educação infantil sob a ótica das professoras. Revista Brasileira de Educação Especial, v. 15, n. 1, p. 121-140, abr.

Miranda, A. A. B. (2008). Educação Especial no Brasil: Desenvolvimento Histórico. Cadernos de História da Educação, v. 7, p. 29-44.

Pelosi, M. B. e Nunes, L. R. D. de P. (2011). A ação conjunta dos profissionais da saúde e da educação na escola inclusiva. Revista de Terapia Ocupacional da Universidade de São Paulo, v. 22, n. 1.

Polonia, A. da C. e Dessen, M. A. (2005). Em busca de uma compreensão das relações entre família e escola. Psicologia Escolar e Educacional, v. 9, n. 2.

Saraiva, L. A. e Wagner, A. (2013). A relação família-escola sob a ótica de professores e pais de crianças que frequentam o Ensino Fundamental. Ensaio: Avaliação e Políticas Públicas em Educação, v. 21, n. 81, p. 739-772.

Schwaber, K. (2004). Agile project management with Scrum. Redmond, Wash: Microsoft Press.

Silberschatz, A., Sundarshan, S. e Korth, H. F. (2012). Sistema de banco de dados. Traducao Daniel Vieira. 6. ed. Rio de Janeiro: Elsevier.

Silva, C. C. B. da, Molero, E. S. da S. e Roman, M. D. (2016). A Interface entre Saúde e Educação: percepções de educadores sobre educação inclusiva. Psicologia Escolar e Educacional, v. 20, n. 1, p. 109-115.

Soares, M. D. S (2004). Comparação entre Metodologias Ágeis e Tradicionais para o Desenvolvimento de Software. INFOCOMP Journal of Computer Science, v. 3, n. 2, p. $8-13$.

Sommerville, I. (2011). Engenharia de Software. Traducão Kalinka Oliveira; Ivan Bosnic. 9. ed. São Paulo: Pearson Prentice Hall.

Stauffer, M. (2019). Laravel: Up \& Running: A Framework for Building Modern PHP Apps. Sebastopol, CA: O'Reilly Media.

Vilaronga, C. A. R. e Mendes, E. G. (2014). Ensino colaborativo para o apoio à inclusão escolar: práticas colaborativas entre os professores. Revista Brasileira de Estudos Pedagógicos, v. 95, n. 239, p. 139-151. 